\title{
DETERMINACIÓN Y DISEÑO DE INDICADORES \\ DE GESTIÓN PARA LA INDUSTRIA \\ DEL CALZADO COMO HERRAMIENTAS \\ PARA EVALUAR LA RENTABILIDAD
}

\author{
INÉS VILLAFANA* \\ https://orcid.org/0000-0003-2236-8247 \\ Universidad de Lima, Perú
}

Recibido: 16 de mayo del 2021 / Aprobado: 5 de julio del 2021

doi: https://doi.org/10.26439/ing.ind2021.n41.5544

RESUMEN: La industria del calzado se encuentra poco desarrollada en el Perú. En Lima existen empresas con diferentes niveles de ventas y con un desarrollo y control de sus procesos distintos (algunos de manera empírica). Con financiamiento del Instituto de Investigación Científica (IDIC) se ha desarrollado esta investigación cualitativa (exploratoria) aplicando entrevistas a expertos (2013) y se ha concluido que el $83,33 \%$ de estas empresas no cuentan con indicadores. Por ello aquí se proponen algunos para las áreas comerciales y de producción.

PALABRAS CLAVES: indicadores de gestión / KPI / industria de calzado / zapatos

IDENTIFICATION AND DESIGN OF PERFORMANCE INDICATORS

IN THE SHOE INDUSTRY AS TOOLS TO EVALUATE PROFITABILITY

ABSTRACT: The shoe industry is underdeveloped in Peru. Within Lima, companies of various levels of profitability manage their businesses in their own way (some empirically). Sponsored by the Scientific Research Institute (IDIC), this qualitative (exploratory) research has been developed by conducting interviews with experts (2013), concluding that $83,33 \%$ of these companies do not have key performance indicators, thus proposing them for both commercial and production areas.

KEYWORDS: performance indicators / KPI's / shoe's industry / shoes

*Correo electrónico: 19900796@aloe.ulima.edu.pe 


\section{INTRODUCCIÓN}

La industria del calzado se desarrolla en el Perú, principalmente, a través de pequeñas y medianas empresas (pymes). Estas empresas de calzado se encuentran concentradas, en una mayor proporción, en Lima y Trujillo (Ministerio de la Producción s. f.). En Lima, las empresas se encuentran ubicadas geográficamente en distintas zonas, como Caquetá, Villa El Salvador, Villa María del Triunfo, San Juan de Miraflores y Lima Centro. Estas empresas también se clasifican por su tamaño; de acuerdo con sus ventas e independientemente del tamaño o ubicación geográfica, todas ellas necesitan llevar controles de sus procesos para poder crecer dentro del sector. Las pymes en el Perú representan aproximadamente el 99,7 \% del total de empresas. La industria del calzado (objeto de estudio) está dominada por las pymes. Según la Sociedad Nacional de Industrias, hacia el año 2011, el 96,7 \% de las empresas productoras de calzado en el Perú eran microempresas, 3,2 \% pequeñas empresas y 0,1 \% medianas y grandes empresas. En Lima está concentrado el mayor número de establecimientos de los fabricantes de calzado, con el 42,2 \% del total (Andina, 2009). Al analizar a estas empresas se puede observar que enfrentan varias dificultades para poder crecer, entre ellas, la informalidad del sector, la reducción del mercado debido a la competencia extranjera, la persistencia de elevadas tasas de interés de los créditos bancarios que limitan la capacidad de inversión, la poca demanda de productos locales por parte del cliente, entre otras (información obtenida de las entrevistas realizadas). Esto hace incluso más difícil la competitividad de esta industria en el Perú.

Una manera de estimular la economía de un país es a través de las exportaciones de sus productos, lo que permite obtener un incremento en la balanza comercial. Esto se puede fomentar a través de la utilización de los tratados de libre comercio. Estos tratados pueden ser aprovechados por las distintas empresas del sector calzado para propiciar la exportación de sus productos con regulaciones beneficiosas entre los países firmantes. Actualmente existen muchos tratados de libre comercio suscritos entre el Perú y otros países, los cuales sirven para fortalecer los lazos de amistad y cooperación y promover la integración económica regional, propiciar la creación de un mercado más amplio y promover el desarrollo económico regional, entre otros (Acomext, 2013).

A continuación se mencionan algunos de los tratados vigentes con Perú que pueden ser aprovechados para promover la exportación del calzado (Acomext, 2013):

- TLC Estados Unidos (Ministerio de Comercio Exterior y Turismo, Acuerdo de promoción comercial Perú-Estados Unidos.

- TLC Canadá (Ministerio de Comercio Exterior y Turismo, Tratado de Libre Comercio Perú-Canadá). 
- $\quad$ TLC Chile (Ministerio de Comercio Exterior y Turismo, Acuerdo de Libre Comercio entre Perú y Chile).

- $\quad$ TLC con la Unión Europea (Ministerio de Comercio Exterior y Turismo, Acuerdo comercial entre Per'y y la Unión Europea).

Por otro lado, los expertos indican que, en el 2013, el número de pymes exportadoras crecería en aproximadamente 50 \%, es decir, más de 1200 nuevos emprendedores se sumarían a la cadena exportadora.

Lima es la ciudad que tiene mayor concentración de empresas de calzado, seguida de Trujillo. La exportación de calzado de Perú hacia los principales países de destino: Chile, Ecuador y Estados Unidos representan un 26,2 \% para el 2018 (Cosavalente, 2019).

Al analizar esta industria, se ha podido identificar algunos problemas que se presentan actualmente en Latinoamérica y en el Perú. Entre ellos tenemos a la globalización del sector, la competitividad internacional (Cosavalente, 2019; Villegas Álvarez y Zapata Gonzáles, 2007), el aumento de la participación del calzado asiático (Cosavalente, 2019), la informalidad y baja inversión tecnológica, el uso de mano de obra poco calificada (Cosavalente, 2019), la aplicación de procesos productivos artesanales (desarrollados por las micro y pequeñas empresas) y la falta de un mayor desarrollo en el diseño del calzado (Díaz y Rodríguez, 2008). Adicionalmente se tiene la dificultad de acceso a tecnología de punta en el Perú porque requiere de una inversión inicial elevada (Soto Brito, 2007), lo que se convierte en una barrera por la falta de capital y/o el difícil acceso al crédito. Asimismo, la nula o poca relación que existe actualmente entre la estandarización de los procesos productivos y su eficiencia en la gestión financiera (Fernández Ávila, 2009), y el acceso a cuero de calidad (dependiendo del poder de negociación de la empresa, esta accede a cuero de primera, segunda o tercera). Debido al bajo costo que tiene en el mercado local, el cuero crudo es altamente demandado en el exterior, lo que hace insuficiente la oferta de dicho insumo (Soto Brito, 2007). Todo esto se ha podido validar con la aplicación de las entrevistas (realizadas en el 2013) a empresas del sector calzado ubicadas en Lima.

Actualmente, el gobierno peruano trata de desarrollar la industria del calzado sobre la base de la promoción de las pymes y el apoyo del Centro de Innovación Tecnológica del Cuero y Calzado e Industrias Conexas (CITECCAL) (Soto Brito, 2007). Se considera importante la creación de CITECCAL y la preocupación del Estado peruano por el desarrollo de este sector; esto debe ser aprovechado por los pequeños productores de calzado; del mismo modo, se debe tener en cuenta la necesidad de formalización de estas empresas.

Asimismo, la inexistencia o poca existencia de indicadores dentro de la industria del calzado impide llevar un adecuado control dentro de las empresas para poder aplicar una mejora continua y crecer en el tiempo; por lo que se incentiva en menor grado la formalización (información obtenida de las entrevistas realizadas). 
Por último, existen estudios anteriores realizados sobre el sector calzado e indicadores varios, a partir de los cuales algunos proponen indicadores macroeconómicos y otros proponen indicadores específicos, como productividad, eficiencia y eficacia, pero de manera general. Se ha podido observar que algunos de estos estudios son un poco antiguos y es necesario actualizar esta información (Flores Konja, 2004; GS1 Chile, 2004).

Por lo tanto, un adecuado diseño de indicadores de gestión podrá favorecer la formalización y el crecimiento de las empresas del sector, posibilitando una mayor productividad e incremento de la rentabilidad del negocio. Para poder lograrlo, se realiza esta investigación.

\section{METODOLOGÍA}

La investigación realizada en el 2013 es una investigación cualitativa (exploratoria). La metodología usada fue, en primer lugar, el estudio de gabinete con la revisión de literatura. Posteriormente, para fines de contraste y validación, se realizó un trabajo de campo mediante un estudio cualitativo exhaustivo a través de la aplicación de entrevistas a algunos dueños y/o gerentes de empresas de calzado de Lima (objeto del estudio), complementado con entrevistas a expertos y académicos de las áreas de producción, calidad y comercio con experiencia en diversos sectores. Las entrevistas se enfocaron principalmente en las áreas comercial y de producción de las empresas del sector calzado (aspectos generales, apertura de la entrevista, procesos de producción, proveedores, área comercial y de ventas, trabajadores, cierre de la entrevista). A partir de un universo de estudio del sector calzado de Lima, conformado por 89 empresas formales, se seleccionó al azar una muestra de seis de ellas de distinto tamaño y ubicación geográfica. Al interior de cada empresa se entrevistó a una o dos personas (el gerente general o propietario del negocio y el gerente de producción y/o gerente comercial) para levantar la información de estas áreas, la cual se complementó con la obtenida por la entrevista aplicada a expertos y académicos de otros sectores de las áreas de producción, calidad y comercio (diez expertos en total), que fueron seleccionadas a criterio de las investigadoras.

Finalmente, con la información obtenida, se procedió a realizar un análisis de contenido y se propusieron indicadores de gestión relacionados con las operaciones críticas de las áreas de producción y comercial del sector calzado que puedan favorecer al incremento de la rentabilidad. Los resultados obtenidos en esta investigación fueron compartidos con las empresas participantes y pueden favorecer a futuros investigadores que se interesen en el sector, así como en el desarrollo de nuevas líneas de investigación. 


\section{RESULTADOS Y DISCUSIÓN}

\section{1 Área de producción y área comercial}

Se deben determinar áreas claves dentro de una empresa del sector calzado para analizar cómo se encuentra actualmente y en qué áreas se deben proponer y aplicar indicadores que controlen y permitan mejorar el desempeño de éstas (en nuestro caso, dentro del área comercial y el área de producción). Esto está sustentado por investigaciones previas desarrolladas por otros investigadores relacionadas de alguna manera con la investigación actual'1.

\section{Área comercial}

Con respecto al área comercial, ésta se puede desagregar en las siguientes actividades: investigación de mercado, desarrollo de producto, marketing, ventas, distribución y/o postventa. Sin embargo, la mayoría de empresas entrevistadas desarrolla el área comercial como un todo y no la tiene desagregada en subáreas o actividades. Igualmente, se están proponiendo indicadores aplicados a algunas de estas áreas.

\section{Área de producción}

Con respecto al área de producción, se procederá a explicar brevemente el proceso de fabricación del calzado (Núcleo Ejecutor Calzado).

La fabricación de calzado consta principalmente de cuatro etapas: corte y desbastado, aparado o costura, montaje o armado y emplantillado, acabado, limpieza y encajado.

a. Área de corte y desbastado

Se reciben los insumos (cuero), se seleccionan y se procede a trazar y cortar las piezas. Posteriormente, se desbastan los bordes (reducción del grosor de las orillas), para un mejor proceso de costura y mejorar la apariencia del corte terminado.

b. Área de aparado o costura

Las partes cortadas se unen mediante costuras y posteriormente, se realiza el montaje sobre la horma.

c. Área de montaje o armado

Se ajusta el cuero cosido sobre la horma y, posteriormente, después de varios procesos toma la forma de un preformado. Al final, se pega la suela y se procede al desmoldado.

1 Fuentes de información (todas las referencias bibliográficas revisadas) 
d) Área de emplantillado, acabado, limpieza y encajado

Se procede a colocar la plantilla y pegar el taco. Posteriormente, se limpia el calzado, se le coloca el pasador (si corresponde), se vaporiza para suavizar las fibras de la piel y evitar agrietamientos o rupturas de la misma; y se realiza un control de calidad. Por último, se procede a colocar el calzado en su respectiva caja.

\subsection{Análisis de resultados}

Las entrevistas aplicadas en las empresas fueron principalmente a dueños o gerentes de producción del sector calzado (la mayoría son dueños de sus negocios). Todas las personas entrevistadas manifestaron contar con experiencia previa en el sector calzado, antes de dedicarse por completo a esta industria. Por otra parte, los entrevistados indicaron que incursionaron en este sector por oportunidad de negocio (consolidación de empresa por inyección de liquidez, existencia de mercado para la venta de los productos y/o oportunidad de crecimiento dentro de la zona).

De acuerdo con la Ley $30056^{2}$, la categoría de las empresas tomando como base su nivel de ventas se ha establecido de la siguiente manera:

- Microempresa: ventas anuales hasta el monto máximo de 150 UIT.

- Pequeña empresa: ventas anuales superiores a 150 UIT y hasta por el monto máximo de 1700 UIT.

- Mediana empresa: ventas anuales superiores a 1700 UIT y hasta el monto máximo de 2300 UIT.

Sobre la base de este nivel de ventas se realizaron las entrevistas en las distintas empresas.

Con respecto a la ubicación geográfica, no existe un lugar definido para las empresas de calzado; están ubicadas en diferentes zonas de Lima. Las empresas en las que se realizaron las entrevistas se encuentran en los distritos de Villa El Salvador, Villa María del Triunfo, San Juan de Miraflores, y en las zonas de la Carretera Central y Lima Centro.

No existe relación entre la cantidad de años que tienen las empresas (desde su constitución) y su tamaño. Una de las empresas con veinticinco años de constitución sigue siendo pequeña; sin embargo, otra empresa con casi la misma cantidad de años actualmente es grande.

2 Ley No. 30056, publicada el 2 de julio del 2013 (Ministerio de Economía y Finanzas y Ministerio de la Producción) 
El 83,33\% de los entrevistados coincide en que la principal dificultad que tuvieron al inicio fue no contar con suficiente capital de trabajo, ya que el acceso al crédito es muy costoso. Asimismo, uno indicó que su principal dificultad fue también el conseguir capital humano con la experiencia necesaria para realizar el trabajo. Por último, otro indicó que tuvieron que lidiar con la devaluación de la época (1980-1990).

Actualmente, la principal limitante para las empresas pequeñas y medianas es el capital de trabajo, ya que las entidades financieras exigen una tasa de interés muy alta por sus préstamos. Algunas empresas cuentan actualmente con los recursos económicos suficientes, pero igual, indican que seguirán invirtiendo en máquinas y tecnología para continuar creciendo.

La manera en que las empresas lograron impulsar su negocio fue a través de apoyo obtenido de diversas entidades como Prompyme, Senati o alguna ONG, utilizando estrategias de nicho de mercado o agrupando el negocio con cadenas de tiendas y, además, consiguiendo más capital.

Las empresas de los entrevistados producen diferentes tipos de calzado (de vestir, sport / casual, niño, escolar, deportivo, de seguridad, etc.); entre ellos, el sport / casual es el más sencillo de fabricar y necesita maquinaria mínima para el proceso.

Cada entrevistado tiene una opinión diferente sobre la etapa del proceso que considera más importante. El $67 \%$ de los entrevistados menciona una etapa y el $33 \%$ coincide en que todas las etapas son igualmente importantes.

Las empresas pequeñas no realizan control de calidad del producto durante el proceso, sino al final del mismo; mayormente realizan el control por observación y de manera empírica. Las empresas medianas y grandes cuentan con controles de calidad establecidos para cada etapa, controlando incluso, con metodología apropiada, los insumos que se utilizan en el proceso productivo.

Solo el $17 \%$ de los entrevistados confirmó que su empresa trabaja actualmente con algunos indicadores de desempeño (KPI); especialmente de producción. Por otro lado, ninguna empresa cuenta actualmente con un sistema de balanced scorecard.

El $83,33 \%$ de los entrevistados coincide en que su cuello de botella es la costura/ aparado o armado/montaje. La costura o aparado demora porque son varias piezas que se tienen que unir, y el armado o montaje demora porque se realiza manualmente.

El 66,67\% de entrevistados coincidieron en que subcontratan o tercerizan el proceso de costura o aparado, principalmente por falta de capacidad de planta y/o porque de esta manera son más eficientes (actualmente hay poca mano de obra calificada y es más conveniente que un tercero especializado les provea el servicio). 
Con respecto a los proveedores, las empresas compran a los mayoristas nacionales; las más grandes inclusive también compran a proveedores del exterior. Todos los entrevistados están satisfechos con sus proveedores, a los que eligen por su seriedad en el cumplimiento, experiencia, referencia, calidad y precio.

Mayormente, las empresas realizan sus ventas dentro del mercado nacional. Algunas han podido inclusive internacionalizarse a través de la exportación.

La gran preocupación de los entrevistados es el crecimiento de las importaciones de calzado chino, que compite a precio bajo. A pesar de esto, para la mayoría de empresas, las ventas han sido más altas con respecto al año 2012.

Finalmente, el 33,33 \% de los entrevistados son miembros de alguna asociación empresarial y también están suscritos a alguna revista del sector calzado.

\subsection{Indicadores de gestión}

Teniendo en cuenta las áreas de producción y comercial, y complementando esta información con lo indicado por las empresas y expertos varios entrevistados, se propone la aplicación de los indicadores siguientes:

Área comercial

a. Cobertura por tipo de producto

Con este indicador se quiere medir la cobertura del mercado atendida por tipo de producto. Así se puede determinar la eficacia de la empresa en la colocación de sus productos en el mercado (resultado logrado / resultado esperado).

$$
\text { Cobertura }=\frac{\text { Ventas reales (unidades modelo } A)}{(\text { Demanda pronosticada (unidades modelo } A)}
$$

Por ejemplo, la demanda pronosticada para enero es de 1000 pares de calzado modelo A. Sin embargo, las ventas logradas en ese mes son de 900 pares de calzado modelo $A$.

Por lo tanto,

$$
\text { Cobertura }=\frac{900 \text { pares } A}{1000 \text { pares } A}=0,9=90 \% \text { de cobertura de mercado }
$$


b. On time performance ${ }^{3}$ (por pedido)

Con este indicador se quiere medir el nivel de cumplimiento a tiempo del pedido (si el pedido se entregó a tiempo al cliente o si se entregó antes o después de la fecha solicitada, expresado en porcentaje).

Si el valor del OTP es:

- Menor al $100 \% \quad \rightarrow \quad$ El pedido se entregó después de la fecha solicitada.

- Igual al $100 \% \quad \rightarrow \quad$ El pedido se entregó en la fecha solicitada.

- Mayor al $100 \% \quad \rightarrow \quad$ El pedido se entregó antes de la fecha solicitada.

c. Días de rezago

Con este indicador se quiere medir el nivel de cumplimiento a tiempo del pedido (si el pedido se entregó en la fecha solicitada o en cuanto tiempo antes o después de la fecha solicitada fue entregado, expresado en unidades de tiempo). En el caso de ser menor a cero, indica que se ha cumplido con la fecha de la entrega del pedido al cliente.

Días de regazo $=[$ Tiempo real - tiempo programado $]($ pedido $N$ ) (días)

d. Fill rate comercial ${ }^{4}$ (por cliente)

Con este indicador se quiere medir el nivel de cumplimiento de entrega de pedidos completos con respecto al total solicitado por el cliente.

$$
\text { Fill rate comercial }=\frac{\text { Número de pedidos entregados completos }}{\text { Número de pedidos totales }}
$$

e. On time delivery ${ }^{5}$ (por cliente)

Con este indicador se quiere medir el nivel de cumplimiento de pedidos entregados a tiempo al cliente con respecto al total de pedidos solicitados por el mismo.

One time delivery (por cliente) $=\frac{\text { Número de pedidos entregados a tiempo }}{\text { Número de pedidos totales solicitados }}$

3 Este indicador se ha considerado a partir de la información revisada: GS1 Chile (2004). Medición indicadores de gestión logísticos (KPI). Guía del usuario.

4 Este indicador se ha considerado de la información revisada: GS1 Chile (2004). Medición indicadores de gestión logísticos (KPI). Guía del usuario.

5 Este indicador se ha considerado de la información revisada: GS1 Chile (2004). Medición indicadores de gestión logísticos (KPI). Guía del usuario. 


\section{f. Eficiencia comercial (por vendedor)}

Con este indicador se quiere medir el porcentaje de cotizaciones que se convierten en pedido por cada vendedor. Se debe tener en cuenta que el número de cotizaciones está dado por una o varias visitas a un mismo cliente. También se puede medir a través del número de pedidos concretados con respecto al total de visitas a los clientes.

Eficiencia comercial

$$
=\frac{\text { Número de pedidos concretados (en un período de tiempo) }}{\text { Número de cotizaciones (en un período de tiempo) }}
$$

\section{Eficiencia comercial}

$$
=\frac{\text { Número de pedidos concretados (en un período de tiempo) }}{\text { Total de visitas a clientes (en un período de tiempo) }}
$$

Este indicador también se puede medir con respecto a las visitas a los clientes que fueron concretadas en ventas.

Eficiencia comercial

$$
=\frac{\text { Número de visitas concretadas en ventas (en un período de tiempo) }}{\text { Total de visitas a clientes (en un período de tiempo) }}
$$

g. Nivel de quejas y reclamos (por cliente)

Con este indicador se quiere medir el número de reclamos hechos por el cliente con respecto al total de pedidos del mismo.

$$
\text { Nivel de quejas y reclamos (por cliente) }=\frac{\text { Número de reclamos (por cliente) }}{\text { Número de pedidos (por cliente) }}
$$

h. Nivel de quejas y reclamos (total)

Con este indicador se quiere medir en qué grado se está dando el número de quejas y reclamos con respecto al total de pedidos (ponderado por clientes). Para esto, se ha clasificado a cada cliente respecto a su grado de importancia (puede ser por las ventas que se facturan con cada uno de ellos). Este indicador debe tender a cero, lo que indicaría que no se tienen quejas ni reclamos.

Nivel de quejas y reclamos (total)

$$
=\sum\left[\frac{\text { Número de reclamos (por cliente) }}{\text { Número de pedidos (por cliente) }} * \text { Calificación de cliente }\right]
$$


Tabla 1

Ejemplo de aplicación: nivel de quejas y reclamos (total)

\begin{tabular}{lcccc}
\hline Cliente & Calificación & $\begin{array}{c}\text { Número } \\
\text { de reclamos }\end{array}$ & $\begin{array}{c}\text { Número de pedidos } \\
\text { totales }\end{array}$ & $\begin{array}{c}\text { Nivel de quejas } \\
\text { (porcentajes) }\end{array}$ \\
\hline Cliente 6 & 0,7 & 4 & 100 & 4,00 \\
Cliente 7 & 0,30 & 1 & 6 & 16,67 \\
Total ponderado: & & 3,10 & 71,8 & 4,31 \\
\hline
\end{tabular}

Elaboración propia

i. Logro por licitación

Con este indicador se quiere medir qué tan eficaz se está siendo al participar en las licitaciones.

$$
\text { Logro por licitación }=\frac{\text { Número de licitaciones ganadas }}{\text { Total de licitaciones presentadas }}
$$

j. Participación de ventas por modelo-color

Con este indicador se quiere medir el porcentaje de participación de cada modelo de calzado que fabrica la empresa con respecto al total de modelos de calzado que fabrica en un periodo. Se da en los casos en que la empresa fabrica varios modelos en un mismo periodo de tiempo. Así se puede determinar qué modelos de calzado son los que se venden en mayor cantidad.

Este indicador también se puede haber medido con respecto al ingreso por ventas que se obtiene por cada modelo de calzado, pero en este estudio no se han considerado indicadores financieros.

$$
\text { Participación en ventas }(\text { modelo }- \text { color })=\frac{\text { Total unidades de modelos } A}{\text { Total unidades de todos los modelos }}
$$

Área de producción

a. On time delivery (pedidos) $^{2}$

Con este indicador se quiere medir el nivel de cumplimiento de pedidos de materia prima recibidos a tiempo por parte de los proveedores con respecto al total de pedidos solicitados por la empresa. También se puede medir en el caso de tercerización del servicio.

6 Este indicador se ha considerado de la información revisada (2004): GS1 Chile. Medición indicadores de gestión logísticos (KPI). Guía del usuario. 
Este indicador no aplica para las compras contra entrega, que se da en los casos de la compra de materia prima por parte de la mayoría de las pymes, ya que ellos les compran directamente a los proveedores ubicados en Caquetá.

On time delivery (pedidos)

$$
=\frac{\text { Número de pedidos recibidos a tiempo }}{\text { Número de pedidos totales solicitados al proveedor o a un tercero }}
$$

Tabla 2

Ejemplo de aplicación: On time delivery (pedidos)

\begin{tabular}{lccc}
\hline Proveedor & $\begin{array}{c}\text { Número de pedidos } \\
\text { a tiempo }\end{array}$ & $\begin{array}{c}\text { Número de pedidos } \\
\text { totales }\end{array}$ & $\begin{array}{c}\text { On time delivery } \\
\text { (porcentajes) }\end{array}$ \\
\hline Proveedor 1 & 0 & 1 & 0,00 \\
Proveedor 2 & 1 & 2 & 50,00 \\
\hline
\end{tabular}

Elaboración propia

b. On time delivery ${ }^{7}$ (unidades)

Con este indicador se quiere medir el nivel de cumplimiento de entrega de unidades por parte de los proveedores de tercerización (en nuestro caso, unidades cosidas o aparadas) con respecto al total de unidades solicitadas por la empresa. También se puede medir teniendo en cuenta si se solicitó la tercerización del servicio por parte de la empresa.

On time delivery (unidades) $=\frac{\text { Número de unidades aparadas recibidas a tiempo }}{\text { Número de unidades totales entregadas a tercerización }}$

c. No conformidad (tercerización o proveedor) y no conformes (unidades o porcentaje) Con este indicador se quiere medir el número de productos no conformes recibidos del proveedor (nacional o extranjero) o del servicio de tercerización, con respecto al total de productos recibidos.

$$
\text { No conformidad (tercerización) }=\frac{\text { Número de unidades no conformes }}{\text { Número de unidades totales recibidas }}
$$

No conformidad (proveedores) $=\frac{\text { Número de mantas de cuero (o avíos) no conformes }}{\text { Número de mantas totales (o avíos) recibidos }}$

7 Este indicador se ha considerado de la información revisada: GS1 Chile (2004). Medición indicadores de gestión logísticos (KPI). Guía del usuario. 
También se puede medir el grado de no conformidad al comparar las unidades no conformes (dentro de cada etapa del proceso) con las unidades totales que deberían salir en cada etapa. Se entiende como no conforme a la unidad que no cumple con el grado de tolerancia establecido dentro de las especificaciones. Este indicador se puede medir tanto en porcentaje como en unidades.

$$
\text { No conformes (porcentaje) }=\frac{\text { Número de no conformes (unidades) }}{\text { Total de unidades ingresadas }}
$$

d. Productividad $^{8}$

Con este indicador se quiere medir la obtención de unidades producidas con respecto a los recursos utilizados.

$$
\text { Productividad }=\frac{\text { unidades productivas }}{\text { recursos utilizados }}
$$

Los recursos utilizados pueden ser:

- Horas-hombre utilizadas para obtener un lote de producción.

- Materia prima utilizada para obtener un lote de producción (en sus respectivas unidades de medida: pies $^{2}$, kilos, etcétera).

- Horas-máquina utilizadas para obtener un lote de producción.

Se recomienda calcular la productividad total y la productividad parcial por cada una de las operaciones del proceso de producción de calzado.

La productividad se mide también en función de los costos. Hay que tener en cuenta que para este trabajo no se ha analizado el área financiera de las empresas.

d. Eficiencia en el uso de materia prima

Con este indicador se quiere medir el nivel de eficiencia en la utilización de la materia prima (manta de cuero u otras) para obtener el producto final (en este caso, todas las piezas de cuero necesarias cortadas para la elaboración del zapato). Se puede considerar para cualquier tipo de materia prima (con sus respectivas unidades).

8 Este indicador se ha considerado de investigaciones previas revisadas: Mena Jaramillo, E.; Paz Martinez, A. (2012). Diagnóstico de la cadena de aprovisionamiento de materias primas e insumos para cuatro eslabones de la industria del cuero y calzado. Universidad de San Buenaventura, Fondo Editorial y Flores Konja, A. (2004). Metodología de gestión para las micro, pequeñas y medianas empresas en Lima metropolitana. Capítulo VIII: Tema de soporte: Indicadores. Universidad Nacional Mayor de San Marcos, Fondo Editorial. 


$$
\text { Productividad }=\frac{\text { pies }^{2} \text { necesarios por lote }}{\text { pies }^{2} \text { utilizados por lote }}
$$

\section{Tabla 2}

Ejemplo de aplicación: eficiencia en el uso de materia prima

\begin{tabular}{cccc}
\hline Corte & $\begin{array}{c}\text { Materia prima necesaria } \\
\left(\text { pie }^{2}\right)\end{array}$ & $\begin{array}{c}\text { Materia prima utilizada } \\
\left(\text { pie }^{2}\right)\end{array}$ & $\begin{array}{c}\text { Eficiencia materia } \\
\text { prima }\end{array}$ \\
\hline Lote 1 & 110 & 130 & $84,62 \%$ \\
\hline
\end{tabular}

\section{Elaboración propia}

Si al $100 \%$ le restamos la eficiencia en el uso de la materia prima, se estaría obteniendo el porcentaje de merma en la operación de corte.

e) Eficacia en las operaciones cuello de botella9

Con este indicador se quiere medir:

- El tiempo real utilizado en la operación de costura o aparado del total de piezas del lote, con respecto al tiempo efectivo o estándar que debería tomar esta operación.

- El tiempo real utilizado en la operación de armado o montaje del total de piezas del lote, con respecto al tiempo efectivo o estándar que debería tomar esta operación.

El tiempo estándar se calcula a través de muestreos con la toma de tiempos y movimientos de la operación cronometrada (incluyendo el tiempo de los suplementos como: tiempo de necesidades personales, básico por fatiga, por postura anormal, entre otros).

$$
\begin{gathered}
\text { Eficacia costura o aparado }=\frac{\text { tiempo de producción efectivo por lote }}{\text { tiempo real de costura por lote }} \\
\text { Eficacia armado o montaje }=\frac{\text { tiempo de producción efectivo por lote }}{\text { tiempo real de armado por lote }}
\end{gathered}
$$

9 Está respaldado por la información revisada: Flores Konja, A. A. Metodología de gestión para las micro, pequeñas y medianas empresas en Lima metropolitana. Capítulo VIII: Tema de soporte: Indicadores y Apaza Meza (2010), M. Balanced scorecard, gerencia estratégica y del valor. 
Con este indicador se quiere medir el grado de cumplimiento de los pedidos con respecto al programa de producción. Se debe tener en cuenta que los pedidos se programan de acuerdo con las órdenes confirmadas de los clientes o a lo pronosticado considerando la data histórica ${ }^{10}$.

$$
\text { Cumplimiento PMP (pedidos) }=\frac{\text { Número de pedidos producidos completos }}{\text { Total de pedidos programados }}
$$

Generalmente, el programa de producción se establece para periodos cortos (semanales).

Asimismo, este indicador se puede medir con respecto al cumplimiento de las unidades programadas (para verificar el cumplimiento con respecto a cada lote de producción).

$$
\text { Cumplimiento PMP (unidades) }=\frac{\text { Número de unidades fabricadas }}{\text { Total de unidades programadas }}
$$

\section{CONCLUSIONES}

- En Lima no hay una zona determinada para las empresas del sector calzado ya que éstas se encuentran establecidas indistintamente en los diferentes distritos. Las zonas más conocidas para el sector calzado son Caquetá y Villa El Salvador.

- Todas coinciden en que la principal dificultad que se les presenta mientras son pequeñas empresas es contar con capital de trabajo suficiente para poder crecer (principalmente inversión en maquinaria), ya que el acceso al crédito es muy costoso. Actualmente muy pocas empresas cuentan con la maquinaria requerida, pero igualmente siguen invirtiendo en tecnología.

- El 83,33 \% de los entrevistados coincide en que el cuello de botella es la costura o aparado y el armado o montaje. El 66,67 \% de las empresas de los entrevistados terceriza el servicio de costura o aparado, ya que así son más eficientes.

- El 83,33 \% de las empresas de los entrevistados no cuenta con indicadores establecidos para controlar sus diferentes áreas y poder determinar si son rentables. Sin embargo, llevan este control de manera empírica.

10 Esta información se sustenta con conceptos teóricos del libro Heizer, J.; Render, B. (2009). Principios de administración de operaciones. Pearson Education. 
- Hay una gran preocupación por la competencia de China, ya que en los últimos años las importaciones de calzado chino se han ido incrementando, restándoles participación de mercado dentro de Lima.

Por lo tanto, con la investigación se han propuesto indicadores de gestión dentro de las áreas de producción y comercial, con lo que, si en un estudio posterior se aplican en una empresa de calzado y se lleva un monitoreo de las mismas, se podrá validar si estos indicadores favorecen la rentabilidad del negocio.

\section{REFRENCIAS}

Acomext. (26 de abril del 2013). Tratado de Libre Comercio para Calzado.

Álvarez, D. C. V., González, H. D. Z., Álvarez, H. F. R., y Carvajal, M. C. V. (2007). Competitividad sectorial internacional: Caso: sector del cuero y del calzado. Entramado, 3(1), 24-49.

Andina - Agencia Peruana de Noticias. (9 de Octubre del 2009). Andina - Agencia Peruana de Noticias. http://www.andina.com.pe/Espanol/noticia-el-967-productorescalzado-peru-son-microempresas-381243.aspx\#.UxZFMPI50Sp

Anthara, I. M. A., y Damayanti W. (2018). Performance Analysis of Supply Chain on Saroo Model Shoes Products Using SCOR Model. IOPConference Series: Materials Science and Engineering, 407(1). https://doi.org/10.1088/1757-899X/407/1/012079

Barcelos da Costa, A. (2010). La industria del calzado del Vale dos Sinos (Brasil): ajuste competitivo de un sector intensivo en mano de obra. Revista CEPAL, 101, 163-79. Naciones Unidas.

Barrenechea, M. (2006) Limitaciones institucionales para el desarrollo de la pequeña y mediana empresa (PYME): el caso de la industria manufacturera de calzado del distrito de Villa el Salvador. Universidad de Lima, Instituto de Investigación Científica.

Barreto, M., Massey, X., y Gutiérrez, M. C. (s. f.). Institucionalidad de las empresas del Boulevard del Cuero en Villa el Salvador. Facultad de Ciencias Sociales, Pontificia Universidad Católica del Perú.

Benítez Montenegro, A. L., Morales Candel, V. A., y Renderos Portillo, M. C. (2012). Plan de comercialización para los micro y pequeños productores de calzado que forman parte de la base de proveedores del programa paquetes escolares, del departamento de San Salvador. Universidad de El Salvador.

Daaboul J., Da Cunha C., Bernard A., y Laroche F. (2011). Design for Mass Customization: Product Variety vs. Process Variety. CIRP Annals - Manufacturing Technology, 60(1), 169-174. https://doi.org/10.1016/j.cirp.2011.03.093 
Chen, S.-L. y Liang, H.-A. (2014). Cause Mapping of Simple and Complex Marketing Strategies. Journal of Business Research, 67(1), 2867-2876. https://doi. org/10.1016/j.jbusres.2012.06.009

Cheng, C.-P. (2014). Embedded Trust and Beyond: The Organizational Network Transformation of Taishang's Shoe Industry in China. En Hwang Wang, J. (Ed.) Border Crossing in Greater China: Production, Community and Identity, pp. 40-60. https://doi.org/10.4324/9781315798325

Cosavalente F. I. (2019). Perú: Situación actual del sector cuero y calzado. BCRP - Sucursal Trujillo.

Cruz Álvarez, L. (2009). Estudio prospectivo en una fábrica mediana de calzado deportivo Horizonte 2005-2013. [Tesis, Universidad de Lima].

Cruz Huamán, J. y Zuñiga Ochoa, C. (2010). Mejora integral de la empresa Industrias Addax E. I. R. L. Universidad de Lima, Seminario de Investigación.

Daaboul J., Bernard A., y Laroche F. (2016). Knowledge Management, Value Chain Modelling and Simulation as Primary Tools for Mass Customization. 2009 IEEE International Technology Management Conference, ICE 2009 https://doi. org/10.1109/ITMC.2009.7461385

Daaboul J., Laroche F., y Bernard A. (2016). Determining the CODP Position by Value Network Modeling and Simulation. 2010 IEEE International Technology Management Conference, ICE 2010. https://doi.org/10.1109/ICE.2010.7476995

Díaz D., L. P., y Rodriguez Tarazona, Y. N. (2008). La cadena productiva del cuero, calzado y marroquinería en el marco internacional. Universidad Industrial de Santander, Facultad de Ciencias Humanas, Escuela de Economía y Administración.

Donaghu M.T., y Barff R. (1990). Nike Just Did It: International Subcontracting and Flexibility in Athletic Footwear Production1. Regional Studies, 24(6), 537-552. https://doi.org/10.1080/00343409012331346204

El Comercio, (13 de Febrero del 2014). BBVA descartó que el dólar alcance los S/.3,0 durante el año. El Comercio. http://elcomercio.pe/economia/peru/bbva-descartoque-dolar-alcance-s30-durante-ano-noticia-1709404.

ElComercio. (14 de Febrero del 2014). La economía peruana creció $5.02 \%$ durante el 2013. El Comercio. http://elcomercio.pe/economia/peru/economia-peruana-crecio-502durante-2013-noticia-1709637.

Fauzi A.R., Ridwan A.Y., y Juliani W. (2019). Supply Chain Performance Measurement System Development for Shoes SME using Subcontract Production Strategy Based on Integrated SCOR-BSC Model. IOP Conference Series: Materials Science and Engineering, 598 (1). https://doi.org/10.1088/1757-899X/598/1/012126 
Fernández Avila, M. L., (2009) Estandarización de los procesos de la producción y su incidencia en la eficiencia de la gestión en la industria del calzado en el Perú. Universidad San Martin de Porres, Fondo Editorial.

Flores Konja, A. A. (2004). Metodología de gestión para la micro, pequeñas y medianas empresas en Lima Metropolitana. Capítulo VIII: Tema de Soporte: Indicadores. [Tesis para optar el grado académico de doctor en Ciencia Contables y Empresariales. Universidad Nacional Mayor de San Marcos].

Foncodes, Ministerio de la Producción, Ministerio de Educación y Sociedad Nacional de Industrial (Núcleo Ejecutor Calzado). (2009). Manual de fabricación de calzado escolar.

Freeman, R. B., y Kleiner M. M. (2005). The Last American Shoe Manufacturers: Decreasing Productivity and Increasing Profits in the Shift from Piece Rates to Continuous Flow Production. Industrial Relations, 44 (2), 307-330. https://doi. org/10.1111/j.0019-8676.2005.00385.x

Gamarra Cuba, R. (2009). Estudio preliminar para la instalación de una fábrica de producción de calzado de cuero de vestir. Universidad de Lima, Seminario de Investigación.

GS1 Chile. (2004). Medición indicadores de gestión logísticos KPI (Key Performance Indicators). Guía del usuario.

Instituto de Investigación Científica (IDIC). https://www.ulima.edu.pe/investigacion

Jaruga, I. (2018). Footwear Industry in Europe and Worldwide [Przemyst obuwniczy w Europie ina świecie]. Przeglad Wlokienniczy, 72(12), 25-27. https://doi.org/10.15199 /60.2018.12.2

Kumar, A., Moktadir M. A., Khan S. A. R., Garza-Reyes J. A., Tyagi M., y Kazançoğlu, Y. (2020). Behavioral Factors on the Adoption of Sustainable Supply Chain Practices. Resources, Conservation and Recycling, 158. https://doi.org/10.1016/j. resconrec.2020.104818

Laoha, C., y Sukto S. (2015). Lean Assessment for Manufacturing of Small and Medium Enterprises: A Case Study of 2 Industrial Groups in Northeast of Thailand. International Business Management, 9(4), 590-595. https://doi.org/10.3923/ ibm.2015.590.595

Marcelo, M.T., Avila G. V., Cruz, M. A., Prado, B. M. y Navarro, M. M. (2016). Process Improvement and Utilization of Machines in the Production Area of a Shoe Manufacturing Company. IEEE International Conference on Industrial Engineering and Engineering Management, 701-705. https://doi.org/10.1109/ IEEM.2016.7797966 
Mena Jaramillo, E. F., y Paz Martínez, A. C. (2012). Diagnóstico de la cadena de aprovisionamiento de materias primas e insumos para cuatro eslabones de la industria del cuero y calzado. Universidad de San Buenaventura, Fondo Editorial.

Ministerio de Economía y Finanzas (MEF). (s/f). https://www.gob.pe/mef

Ministerio de la Producción. (s. f.). https://www.gob.pe/produce

Nafizah, U. Y. y Tiara, A. R. (2018). Value Stream Mapping in Indonesia's Shoe Industry: An Alternative Tool to Map Innovation Ecosystem. International Journal of Technological Learning, Innovation and Development, 10(2), 113-130. https://doi. org/10.1504/IJTLID.2018.093159

Núcleo ejecutor calzado. (s. f.). Manual de fabricación escolar.

Parker, P. M. (2011). The 2009 Import and Export Market for Sports Footwear in Peru. Country Trade Report. 16. Business Source Elite de EBSCO.

Tello, M. D. (2012). Productividad total factorial en el sector manufacturero del Perú: 2002 -2007. Economía, 70, 103-141. ProQuest.

Tito Huaman, P. L. (2012). Gestión por competencias y productividad laboral en empresas del sector confección de calzado de Lima metropolitana. Universidad Nacional Mayor de San Marcos, Fondo Editorial.

Sociedad Nacional de Industrias. (2013). https://sni.org.pe

Soto Brito, H. (2007). La competitividad de la industria del calzado en el Perú y sus proyecciones en el mediano plazo (caso pyme Tobbex International y el papel de Citeccal). Universidad Nacional Mayor de San Marcos, Fondo Editorial.

Sri Rejeki, Y., Rahman As'ad, N., y Achiraeniwati, E. (2014). Improvement of Work System with Ergonomic Approach of Domestic Shoe Industry in Cibaduyut Bandung. Applied Mechanics and Materials, 606, 247-251. https://doi.org/10.4028/www. scientific.net/AMM.606.247

Velasco Rivera, S. (s. f.). Como se puede incursionar en una pequeña empresa de calzado al comercio exterior enfrentándose a la competitividad. Universidad Vasco de Quiroga, Fondo Editorial.

Villegas Álvarez, D. C., y Zapata González, H. D.; Auxiliares de investigación: Rodríguez Álvarez, Héctor Fabio, Vega Carvajal, María Cristina (enero-junio 2007). Competitividad sectorial internacional. Caso: sector del cuero y del calzado. 
